\title{
Elevation of Oxidative Stress Indicators in a Pilot Study of Plasma Following Traumatic Brain Injury
}

Alison Halstrom, BSc ${ }^{\mathrm{a}}$, Ellen MacDonald, $\mathrm{RN}^{\mathrm{b}, \mathrm{c}}$, Claire Neil, BSc ${ }^{\mathrm{c}}$, Glenn Arendts, MBBS FACEM ${ }^{\text {b,c }}$, Daniel Fatovich, FACEM, Phb ${ }^{2, c}$, Melinda Fitzgerald, A/Prof ${ }^{a}$

${ }^{a}$ Experimental and Regenerative Neurosciences, School of Animal Biology, The University of Western Australia, Perth 6009, Western Australia, Australia

${ }^{b}$ Emergency Medicine, Royal Perth Hospital, The University of Western Australia, Perth, Western Australia, Australia

${ }^{c}$ Centre for Clinical Research in Emergency Medicine, Harry Perkins Institute of Medical Research

Corresponding author:

Melinda Fitzgerald, Assoc/Prof

lindy.fitzgerald@uwa.edu.au

Alison Halstrom, BSc

20947223@student.uwa.edu.au

Ellen MacDonald, Clinical Nurse Manager Emergency Research

ellen.macdonald@uwa.edu.au

Claire Neil, BSc, Grad RA

claire.neil@uwa.edu.au 
Glenn Arendts, MBBS MMed FACEM

glenn.arendts@uwa.edu.au

Daniel Fatovich, MBBS FACEM PhD,

daniel.fatovich@health.wa.gov.au

MF is supported by a National Health and Medical Research Council, Australia, Career Development Fellowship (APP1087114). 


\begin{abstract}
Traumatic brain injury (TBI) encompasses a broad range of injury mechanisms and severity. A detailed determination of TBI severity can be a complex challenge, with current clinical tools sometimes insufficient to tailor a clinical response to a spectrum of patient needs. Blood biomarkers of TBI may supplement clinical assessments but currently available biomarkers have limited sensitivity and specificity. While oxidative stress is known to feature in damage mechanisms following TBI, investigation of blood biomarkers of oxidative stress has been limited. This exploratory pilot study of a subset of 18 trauma patients with TBI of varying severity, quantifies circulating concentrations of the structural damage indicators S100b, and myelin basic protein (MBP), and the biomarkers of oxidative stress hydroxynonenal (HNE), malondialdehyde (MDA), carboxy-methyl-lysine (CML), and 8hydroxy-2'-deoxy-guanosine (8-OHDG). Significant increases in circulating S100b, MBP, and HNE were observed in TBI patient samples compared to 8 uninjured controls, and there was a significant decrease in CML. This small exploratory study supports the current literature on S100b and MBP elevation in TBI, and reveals potential for the use of peripheral oxidative stress markers to assist in determination of TBI severity. Further investigation is required to validate results and confirm trends.
\end{abstract}

Keywords: biomarkers; traumatic brain injury; oxidative stress; hydroxynonenal 


\section{Introduction}

Traumatic brain injury (TBI) is a major global public health burden, accounting for over 1.7 million emergency department (ED) presentations annually in the United States alone. ${ }^{1}$ TBI's are commonly classified as mild, moderate, or severe. Classification is based primarily on a patient's Glasgow Coma Scale (GCS), which gives a combined measure of eye, verbal, and motor response to stimuli. ${ }^{2}$ Moderate and severe TBI are typically associated with gross neural pathologies such as haematoma and oedema, a need for extensive intervention, and significant morbidity in survivors. ${ }^{3,4}$ Primary responses to moderate and severe TBI are often dictated by the results of imaging analysis. While moderate and severe TBI are of great concern, as many as $80 \%$ of all TBI's are classified as 'mild'. ${ }^{4}$ Mild TBI (mTBI) patients display no gross neural pathologies using standard imaging methods, and have no immediate threat to life. However, many patients experience debilitating chronic symptoms which can impede their ability to return to work, social functioning, and psychological health for months to years after even a single mTBI. ${ }^{3,5,6}$ Limitations of current tools to guide clinical decisions and predict patient outcome, have prompted the search for biomarkers that can be used to detect, quantify and differentiate underlying cellular damage in TBI.

Primary mechanical injury following TBI leads to cascades of secondary neurophysiological damage, resulting in chronic cognitive, motor, and emotional deficits, potentially revealed in a range of downstream biomarkers. ${ }^{3,7}$ Animal models of mild, moderate and severe TBI, along with behavioural, imaging, and histopathological studies in humans, have revealed the progressive and complex nature of secondary degenerative pathways associated with different TBI severity. Biomarkers of current interest represent a range of these neuropathological mechanisms. Damage to structural proteins of both neurons and glia occur with the initial impact, and continues with secondary injury processes. ${ }^{3,7}$ 
Calcium binding S100 protein beta (S100b), myelin basic protein (MBP), neuron specific enolase (NSE), and calpain-derived $\alpha$ II-spectrin N-terminal fragment (SNTF) are some of the markers elevated in cerebrospinal fluid and blood due to mechanical injury to cells and resulting ionic imbalance. ${ }^{8,9} \mathrm{~S} 100 \mathrm{~b}$ in particular has been identified as a blood biomarker, which may correlate to injury severity and outcome in moderate to severe TBI patients. ${ }^{9,10}$ While many biomarkers of TBI show promise, each has its limitations. S100b concentrations can be significantly affected by extra-cerebral poly-trauma. ${ }^{11}$ Inflammatory markers are elevated peripherally for days to weeks after TBI, making them potentially valuable in gauging recovery and experimental treatment efficacy. However, blood concentrations have not been found to correlate consistently with TBI severity or outcome. ${ }^{9,12}$

Oxidative stress occurs as a consequence of multiple pathological processes active in TBI, and oxidised products are appealing as peripheral blood markers for generalised cellular, axonal, lipid, protein or DNA damage. ${ }^{13}$ However to date, few oxidative stress indicators have been investigated for utility as blood biomarkers in humans across a range of TBI severities. Malondialdehyde (MDA) is one of the best characterised indicators of oxidative damage to polyunsaturated fatty acids and may serve as a predictor of early mortality in severe TBI. ${ }^{14}$ Hydroxynonenal (HNE) and carboxy-methyl lysine (CML) are also markers of lipid peroxidation, and are peripherally elevated in a range of central nervous system injuries. ${ }^{15-17}$ The base adduct 8-hydroxy-2'-deoxyguanosine (8-OHDG) is formed following exposure of chromatin to free radicals, and is used as a marker of DNA oxidation. ${ }^{18}$ 8-OHDG is known to be elevated in animal models of TBI. ${ }^{19}$ Each of these markers of oxidative stress have potential to become useful in the assessment and management of TBI patients. However more information is required to determine whether circulating levels are elevated in a severity dependant manner in TBI patients. This small exploratory study assesses a range of oxidative stress indicators in blood from patients with TBIs of varying severity. 


\section{Materials and Methods}

\section{Study Design}

Participants were enrolled in a prospective, observational Critical Illness and Shock Study (CISS) between March 2010 and January 2013, recruited from the Emergency Department of Royal Perth Hospital (RPH). ${ }^{20}$ CISS is a prospective study of patients presenting to the Emergency Department with critical illness or injury that compromise the cardiovascular and/or respiratory systems, focussing on the acute immune response to anaphylaxis, septic shock, traumatic brain injury and post-cardiac arrest. Ethics approval was obtained through the RPH human research ethics committee (HREC RPH 2015-062). Because the need for emergency care took priority, waiver of initial consent was approved under the provision of paragraph 2.3.6 of the National Health and Medical Research Council Ethical Conduct guidelines. ${ }^{21}$ Consent was requested from patients or next of kin as appropriate, and patients were given the option of complete withdrawal from the study. A range of blood samples were collected from participating patients, including blood for separation into plasma in ethylene-diamine-tetra-acetic acid (EDTA) tubes. These were stored at $-80^{\circ} \mathrm{C}$ immediately after processing in the purpose built wet lab in the Emergency Department.

The current study describes outcomes from patients in the CISS with: 1) sufficient volumes of EDTA plasma available for use; 2) head injury reported as the principal component of trauma; 3) plasma samples collected within seven hours of injury (time selected based upon available samples and the desire to sample for potentially transient oxidative stress indicators soon after patient presentation); 4) minimal poly-trauma (New Injury Severity Score (NISS) head fraction $>73 \%$, value selected to reflect predominantly head injury for the cohort); and 5) minimal pre-existing conditions (Charlson comorbidity index $\leq 1){ }^{22}$ A single time point of sampling, as soon after patient presentation as possible, 
was chosen in order to allow determination of suitability of biomarkers for less severe TBI when only a single blood sample would be likely to be taken. Plasma samples were analysed in duplicate, together with 8 age and sex matched control samples, which were subjected to the same storage conditions and handling as patient samples. TBI patients were divided into severity groups based on GCS recorded at time of presentation at the Emergency Department, or at scene of injury if sedated for clinical care. Mild injuries included GCS from 13 to 15, moderate between 8 and 13, and a score of less than 8 was classified as severe TBI.

\section{Biomarker Assessment}

Plasma samples were analysed using commercially available enzyme linked immunosorbent assay (ELISA) kits to determine concentrations of selected biomarkers. Quantitative commercial kit analyses allowed rapid determination of analyte concentrations, with well characterised detection sensitivities and selectivities. Biomarkers analysed were; HNE Histone, Lysine, and Cysteine adducts (OxiSelect HNE adduct competitive ELISA kit, STA-838, Cell Biolabs, San Diego), MDA (Thiobarbituric acid reactive species trichloroacetic acid method assay kit, 700870, Cayman Chemicals, Ann Arbour), S100b (Human S100b ELISA, KT-435, Kamiya Biochemical Company, Seattle), MBP (Human myelin basic protein ELISA kit, MyBioSource, \#261463), 8-OHDG (highly sensitive 8OHdG check ELISA, Japan Institute for the Control of Ageing, Shizuoka), and CML (OxiSelect $\mathrm{N}^{\varepsilon}$-(carboxymethyl) lysine competitive ELISA kit, STA-816 Cell Biolabs, San Diego). All analytical procedures were performed according to manufacturer's instructions, and analyte concentrations were determined with reference to standard curves, generated from standards assessed on the same 96 well plate as patient and control samples. Each sample was assessed in duplicate within an assay, with absorbance measured using an EnSpire multimode plate reader (Perkin Elmer, product no. 2300). 


\section{Data Analysis}

All biomarker concentrations were calculated as mean values of duplicate analysis for each patient sample. Outliers, defined as greater than two standard deviations from the mean of all samples of a particular biomarker, were excluded. Concentration distribution of biomarkers in plasma samples from TBI patients were first assessed for normality, then compared to controls, and significance determined for each biomarker across all samples using T-Tests. Severity groups were then compared to each other and controls by single factor analysis of variance (ANOVA), applying Sheffe's post hoc if variances were equal, or Games-Howell if Levene’s test reported a significant difference in variances which could not be corrected by simple data transformations. The method of least squares was applied to determine the linear correlation coefficients between neurological injury severity and biomarker concentration with significance set at $p<0.05$. Pearson's $r$ is reported. Linear regression analysis was used to investigate relationships between biomarker levels, and clinical and demographic details (e.g. age, months of sample storage, poly trauma severity etc.), which varied greatly within the sample set. Partial predictors accounting for more than $5 \%$ of variation in any single outcome are reported. 


\section{Results}

\section{Biomarkers of Structural Proteins}

Eighteen patients met the inclusion criteria. 14 (78\%) patients were male, with a mean age of 34 years $(S D=17.6)$. Details of patients from whom samples were obtained are outlined in Table 1. Concentrations of the structural damage marker S100b required $\log _{10}$ transformation to achieve normal distribution. Variance of data distributions were unequal between TBI severity groups and controls, even following $\log _{10}$ transformation (Levene’s F = 6.288, $\mathrm{p}=0.003$ ). Concentrations of S100b were significantly elevated in grouped samples from patients with TBI of all severities, compared to controls $(\mathrm{P}=0.017, \mathrm{DF}=24)$. Power analysis of the t-test between control and 'all TBI' group conducted post hoc reported a large effect size with moderate power (Cohen's $d=1.06$, power $[1-\beta]=0.645$ ). Comparison of outcomes from subsets of TBI patients with injuries of varying severity and control were conducted using one way ANOVA, with Games-Howell post hoc corrections for all possible multiple comparisons. ANOVA reached significance $(\mathrm{p}=0.003, \mathrm{DF}=25)$, and post hoc analyses revealed a significant difference only between severe TBI patient S100b levels and controls $(p=0.007, D F=16)$. ANOVA comparing TBI severity groups to each other and controls achieved overall only a moderate effect with very weak power of analysis (Cohen's f $=0.319$, power $[1-\beta]=0.178)$, indicating the need for caution in interpreting these results. Pearson's correlation analysis demonstrated a significant moderate severity dependent decrease in plasma levels of S100b with higher GCS $(r=-0.399, p=0.043)$, however when the control group was removed from this analysis significance was not maintained $(r=0.117$, $p=0.643$ ). Linear regression analysis revealed significant effects of age, and NISS (adjusted $r^{2}=0.374$, and 0.197 respectively) (Fig. $1 \mathrm{~A}$ ). 
MBP concentrations required square root transformation to achieve normality and were found to have equal variance (Levene's $\mathrm{F}=1.11, \mathrm{p}=0.367$ ). MBP concentrations were also elevated in patients with TBI compared to controls $(\mathrm{P}=0.030, \mathrm{DF}=22)$. However one way ANOVA comparing controls to subsets of patients with TBI of varying severities did not reveal significant differences in plasma $\mathrm{MBP}$ concentration $(\mathrm{p}=0.127, \mathrm{DF}=23)$ (Fig. 1B). None of the independent variables assessed for interactions had a significant effect.

\section{Biomarkers of Oxidative Stress}

The concentration of the lipid peroxidation indicator HNE was normally distributed, however a $\log _{10}$ transformation was used in order to achieve equal variance (Levene's $\mathrm{F}=$ 1.16, $\mathrm{p}=0.350)$. HNE was found to be significantly elevated in TBI patients compared to controls $(\mathrm{P}=0.035, \mathrm{DF}=21)$ (Fig. 1C). Power analysis of the t-test between control and 'all TBI' group conducted post hoc reported a large effect size with moderate power (Cohen's d = 1.04 , power $[1-\beta]=0.742)$. A more weakly powered $($ Cohen’s $f=0.504$, power $[1-\beta]=0.422)$ ANOVA with Sheffe's post hoc analysis revealed a significant difference between control and severe TBI patient HNE concentrations $(\mathrm{p}=0.050, \mathrm{DF}=16)$. Note that only three patient samples were available for moderate TBI for this outcome measure, making it difficult to draw conclusions for this group. Nevertheless, a moderately strong negative linear correlation

of HNE concentration to GCS was found within TBI patients' samples when the control group was included in analysis $(r=-0.576, \mathrm{p}=0.004)$, but was lost when investigating TBI patients only $(r=-0.441, p=0.128)$. Partial predictors of HNE plasma concentrations determined by regression analysis included; months of $-80^{\circ} \mathrm{C}$ storage (adjusted $r^{2}=0.385$ ), gender (adjusted $r^{2}=0.244$ ), and delay to collection post injury (adjusted $r^{2}=0.180$ ).

MDA concentrations were not found to be significantly elevated when all TBI patients were compared to controls by t-test $(\mathrm{p}=0.199, \mathrm{DF}=23$ ) (Fig. 1D). One way ANOVA 
showed no difference between any TBI severity groups and controls $(p=0.298, D F=23)$. MDA concentrations displayed no significant correlation to GCS with $(r=-0.398, p=0.060)$, or without $(\mathrm{r}=-0.328, \mathrm{p}=0.215)$ inclusion of control group in correlation analysis. No relationships of circulating MDA to any available predictive variables were identified.

Unexpectedly, CML concentrations were found to be significantly lower in TBI samples than in control $(P=0.0024, D F=23$, Cohen's $d=1.21$, power $[1-\beta]=0.865)$ (Fig. 1E). One way ANOVA with Scheffe's post hoc corrections revealed that plasma CML concentrations were significantly lower in the subset of severe TBI patients compared to controls $(p=0.044$, $D F=15$, Cohen's $f=1.61$, power $[1-\beta]=0.999)$. None of the independent variables assessed for interacting relationships were seen to have a significant effect, however a significant moderate positive correlation of CML to GCS was identified when control group was included in analysis $(\mathrm{r}=0.481, \mathrm{p}=0.015)$, but not in TBI patients alone $(\mathrm{r}=0.275, \mathrm{p}=0.391)$.

8-OHdG concentrations were found to be below the lower limit of detection of the ELISA kit $(0.0625 \mathrm{ng} / \mathrm{mL})$ in $\geq 50 \%$ of participant samples in each group. Concentrations below the lower limit were converted to a set value of half of the lowest reliable concentration, resulting in a distribution that was not normal and that had unequal variance. This could not be corrected by simple transformations, so no statistical analyses were conducted. 


\section{Discussion}

This small 'pilot style' study suggests that oxidative stress biomarkers are worthy of further investigation. Of interest, the lipid peroxidation biomarker HNE was consistently low in controls and elevated in TBI samples. Furthermore, there was a trend to a severity dependent increase in HNE with TBI. The decrease in CML levels with severe TBI is also a novel finding warranting further investigation. Quantification of S100b revealed a trend to severity dependent elevation in TBI patient samples, consistent with current literature. ${ }^{9,10}$ While many biomarkers of TBI have been investigated, few are sensitive to injury severity ie mild, moderate or severe. ${ }^{9}$ Our results suggest that HNE, and perhaps CML may be valuable additions to a suite of blood biomarkers, including S100b. However, the small sample size of the current study, combined with the variability in biomarker concentrations indicates the need for further investigation of oxidative stress biomarkers as indicators of injury severity following TBI.

HNE concentrations were moderately and weakly partially predicted by storage time, and delay from injury to sample collection respectively. This may reflect a short half-life of this biomarker both in vivo, and in vitro. While a degradation effect is difficult to assess in a small study such as this, a consistent degradation rate in vivo, once fully characterised, may prove useful in determining time of injury in uncertain cases. The reported weak elevation in female vs. male samples may be an artefact of small sample size, and skewed sex ratios (78\% male). These variables were found to account for some of the variability in biomarker concentration, however further validation of these relationships is required.

MDA concentrations were highly variable across all groups, as expected of this generalised lipid peroxidation marker. MDA is known to be elevated due to a wide range of demographic and lifestyle variables, such as age, fitness, and smoking status. ${ }^{23}$ To our 
knowledge, a decrease in CML concentration with central nervous system injury has not previously been observed. CML has been reported to be elevated in optic nerves vulnerable to secondary degeneration in rats, and in patient serum for retinopathy; ${ }^{16,17}$ although the observed TBI severity dependent decrease in sample concentration has not previously been reported. 8-OHDG concentrations were much lower than expected in all samples. It is possible that this DNA oxidation marker decays with extended storage, and investigation of fresh samples or the use of a more sensitive quantification assay would be of interest.

S100b levels were consistently low in control samples, a valuable trait in any biomarker. However, elevation with injury was not significantly related to TBI severity. Age and NISS were found to be partial predictors of circulating S100b concentration. Higher levels in older TBI patient samples support findings that protective mechanisms minimising tissue damage may be less efficient in older trauma patients. ${ }^{6}$ Elevation with NISS maintains the current view that S100b is not sufficiently neuro-specific to be applied as a TBI marker independently. ${ }^{11} \mathrm{MBP}$, which due to its role in myelin is more neuro-specific than S100b, was more highly variable than S100b in control participants, only seen to be significantly increased when comparing control to all TBI patient samples.

Twenty percent of patients with mTBI develop long term sequelae. ${ }^{3,5,6}$ If a biomarker or set of biomarkers reliably identified these cases at the time of initial assessment, it would be possible to focus on neurorehabilitation strategies that could improve patient outcomes. Furthermore, in current clinical practice a decision to perform neuroimaging in head injury is guided by decision aids that are entirely clinical. ${ }^{25}$ These aids all understandably sacrifice specificity for sensitivity, so still result in a large amount of redundant imaging. A reliable biomarker could refine these decision aids and help define a larger group that does not require neuroimaging. For the more severe TBIs, a biomarker that provided short term and/or long 
term prognostic information may help guide clinical care, such as the intensity of monitoring for deterioration.

This exploratory study of TBI biomarkers in a subset of trauma patients has revealed potential for the use of peripheral oxidative stress markers to assist in determination of TBI severity. While patient numbers are low, the results support the value of further investigation. Validation of results using a specifically recruited cohort, larger sample size of both injured and a range of control groups, stronger quantification of demographic and clinical details, and integration of clinical and experimental imaging will add significant strength to current findings.

\section{Acknowledgements}

MF is supported by a National Health and Medical Research Council, Australia, Career Development Fellowship (APP1087114).

\section{Author Disclosure Statement}

No competing financial interests exist. 


\section{References}

1. Coronado, V.G., McGuire, L.C., Sarmiento, K., Bell, J., Lionbarger, M.R., Jones, C.D., Geller, A.I., Khoury, N., and Xu, L. (2012). Trends in traumatic brain injury in the U.S. and the public health response: 1995-2009. J. Safety Res. 43, 299-307.

2. Teasdale, G., and Jennett, B. (1971). Assessment of coma and impaired consciousness: A practical scale. Lancet 3, 842-266.

3. Bramlett, H.M., and Dietrich, W.D. (2014). Long-term consequences of traumatic brain injury: Current status of potential mechanisms of injury and neurological outcomes. J. Neurotrauma 32, 1834-1848.

4. Helps, Y., Henley, G., and Harrison, J. (2008). Hospital separations due to traumatic brain injury, Australia 2004-05. Inj. Res. Stat. Ser. 45.

5. $\quad$ Bruce, E.D., Konda, S., Dean, D.D., Wang, E.W., Huang, J.H., and Little, D.M. (2015). Neuroimaging and traumatic brain injury: State of the field and voids in translational knowledge. Mol. Cell. Neurosci. 66, 103-113.

6. Johnson, V.E., Stewart, J.E., Begbie, F.D., Trojanowski, J.Q., Smith, D.H., and Stewart, W. (2013). Inflammation and white matter degeneration persist for years after a single traumatic brain injury. Brain 136, 28-42.

7. Blennow, K., Hardy, J., and Zetterberg, H. (2012). The neuropathology and neurobiology of traumatic brain injury. Neuron 76, 886-899.

8. Siman, R., Shahim, P., Tegner, Y., Blennow, K., Zetterberg, H., and Smith, D.H. (2014). Serum SNTF increses in concussed professional ice hockey players and relates to the severity of post-concussion symptoms. J. Neurotrauma 32, 1-33.

9. Zetterberg, H., Smith, D.H., and Blennow, K. (2013). Biomarkers of mild traumatic 
brain injury in cerebrospinal fluid and blood. Nat. Rev. Neurol. 9, 201-10.

10. Thelin, E.P., Johannesson, L., Nelson, D., and Bellander, B.M. (2013). S100B Is an important outcome predictor in traumatic brain injury. J. Neurotrauma 30, 519-528.

11. Ohrt-Nissen, S., Friis-Hansen, L., Dahl, B., Stensballe, J., Romner, B., and Rasmussen, L.S. (2011). How does extracerebral trauma affect the clinical value of S100B measurements? Emerg. Med. J. 28, 941-4.

12. Hergenroeder, G.W., Moore, A.N., McCoy, J.P., Samsel, L., Ward, N.H., Clifton, G.L., and Dash, P.K. (2010). Serum IL-6: a candidate biomarker for intracranial pressure elevation following isolated traumatic brain injury. J. Neuroinflammation 7.

13. Ansari, M.A., Roberts, K.N., and Scheff, S.W. (2008). Oxidative stress and modification of synaptic proteins in hippocampus after traumatic brain injury. Free Radic. Biol. Med. 45, 443-452.

14. Hohl, A., Gullo, J.D.S., Silva, C.C.P., Bertotti, M.M., Felisberto, F., Nunes, J.C., de Souza, B., Petronilho, F., Soares, F.M.S., Prediger, R.D.S., Dal-Pizzol, F., Linhares, M.N., and Walz, R. (2012). Plasma levels of oxidative stress biomarkers and hospital mortality in severe head injury: A multivariate analysis. J. Crit. Care 27, 523.e11523.e19.

15. Lee, W.C., Wong, H.Y., Chai, Y.Y., Shi, C.W., Amino, N., Kikuchi, S., and Huang, S.H. (2012). Lipid peroxidation dysregulation in ischemic stroke: Plasma 4-HNE as a potential biomarker? Biochem. Biophys. Res. Commun. 425, 842-847.

16. Szymanski, C.R., Chiha, W., Morellini, N., Cummins, N., Bartlett, C.A., O’Hare Doig, R.L., Savigni, D.L., Payne, S.C., Harvey, A.R., Dunlop, S.A., and Fitzgerald, M. (2013). Paranode abnormalities and oxidative stress in optic nerve vulnerable to secondary degeneration: Modulation by 670 nm light treatment. PLoS One 8, e66448. 
17. Boehm, B.O., Schilling, S., Rosinger, S., Lang, G.E., Lang, G.K., Kientsch-Engel, R., and Stahl, P. (2004). Elevated serum levels of N(epsilon)-carboxymethyl-lysine, an advanced glycation end product, are associated with proliferative diabetic retinopathy and macular oedema. Diabetologia 47, 1376-1379.

18. Kasai, H. (1997). Analysis of a form of oxidative DNA damage, 8-hydroxy-2'deoxyguanosine, as a marker of cellular oxidative stress during carcinogenesis. Mutat. Res. 387, 147-163.

19. Mendez, D.R., Cherian, L., Moore, N., Arora, T., Liu, P.K., and Robertson, C.S. (2004). Oxidative DNA lesions in a rodent model of traumatic brain injury. J. Trauma 56, 6-11.

20. Arendts, G., Stone, S.F., Fatovich, D.M., van Eeden, P., MacDonald, E., and Brown, S.G. a. (2012). Critical illness in the emergency department: Lessons learnt from the first 12 months of enrolments in the Critical Illness and Shock Study. EMA - Emerg. Med. Australas. 24, 31-36.

21. National Health and Medical Research Council, Australian Research Council, and Australian Vice-Chancellors’ Committee. (2007). National Statement on Ethical Conduct National Statement on Ethical Conduct in Human Research.

22. Bar, B., and Hemphill, J.C. (2011). Charlson comorbidity index adjustment in intracerebral hemorrhage. Stroke 42, 2944-2946.

23. Nielsen, F., Mikkelsen, B.B., Nielsen, J.B., Andersen, H.R., and Grandjean, P. (1997). Plasma malondialdehyde as biomarker for oxidative stress: Reference interval and effects of life-style factors. Clin. Chem. 43, 1209-1214.

24. Menascu, S., Tshechmer, S.M., and Rumeny, G. (2010). Serum biochemical markers for brain damage in children with emphasis on mild head injury. Pediatr. Neurosurg. 
46, 82-88.

25. Ro, Y.S., Shin, S. Do, Holmes, J.F., Song, K.J., Park, J.O., Cho, J.S., Lee, S.C., Kim, S.C., Hong, K.J., Park, C.B., Cha, W.C., Lee, E.J., Kim, Y.J., Ahn, K.O., and Ong, M.E.H. (2011). Comparison of clinical performance of cranial computed tomography rules in patients with minor head injury: A multicenter prospective study. Acad. Emerg. Med. 18, 597-604. 


\section{Figure Legend}

Figure 1: Biomarker concentrations determined by ELISA analysis of TBI patient samples collected within seven hours of injury, and controls. Mean and standard error are displayed for controls, mild, moderate and severe TBI, and all TBI. Severity groups are based on GCS, such that a score of 13 to 15 is mild, 8 to 13 is moderate, and less than 8 classified as severe. Biomarkers assessed: A) calcium binding protein S100 beta, B) myelin basic protein, C) hydroxynonenal adducts, D) malondialdehyde, and E) carboxy-methyl lysine. *denotes significant difference from controls $(\mathrm{P}<0.05)$ when comparing all TBI to controls via T-test. ${ }^{+}$indicates significant difference of a TBI severity subset from control via ANOVA and post hoc test $(\mathrm{P}<0.05)$. 
Table 1

\begin{tabular}{|c|c|c|c|c|}
\hline & \multicolumn{4}{|c|}{ TBI Severity Separations } \\
\hline & Mild & Moderate & Severe & Control \\
\hline $\mathbf{N}$ & 4 & 5 & 9 & 8 \\
\hline Mean age (SD) & $37(20)$ & $39.4(26)$ & $29.8(11)$ & $33.8(12)$ \\
\hline $\mathrm{N}$ male & 2 & 3 & 9 & 6 \\
\hline \multirow[t]{2}{*}{ Median NISS head prop (IQR) } & 14.5 (8.9- & $48(28.5-$ & 33.9 (31.4- & \\
\hline & 20.1) & 67.59) & 36.4) & \\
\hline Mean delay to presentation, min (SD) & $162.5(81)$ & $210(77)$ & $193.9(143)$ & \\
\hline Median ICU days (IQR) & $0(1)$ & $13(12)$ & $5(4)$ & \\
\hline $\mathbf{N}$ deceased & - & 1 & 2 & \\
\hline \multicolumn{5}{|l|}{ GROSS CRANIAL PATHOLOGY } \\
\hline Skull fracture & - & 4 & 6 & \\
\hline Cortical contusion & 2 & 5 & 9 & \\
\hline Intra cranial haemorrhage/ & - & 4 & 7 & \\
\hline \multicolumn{5}{|l|}{ haematoma } \\
\hline Cerebral oedema & - & 1 & 4 & \\
\hline \multicolumn{5}{|l|}{ POLY- TRAUMA } \\
\hline Lacerations (external) & 2 & 3 & 5 & \\
\hline Lacerations (internal) & 1 & - & 1 & \\
\hline Bone fracture (non-skull) & 2 & 3 & 7 & \\
\hline \multicolumn{5}{|l|}{ CLINICAL INTERVENTION } \\
\hline Intubation & - & 5 & 8 & \\
\hline ICP monitoring & - & 2 & 5 & \\
\hline Craniectomy & - & 1 & 3 & \\
\hline
\end{tabular}

Table 1: Demographic information (mean with standard deviation (SD), or median with inter quartile range (IQR)), and clinical injury details of patients with TBI and controls. TBI patients were a subset of the Critical Illness and Shock Study (CISS) as outlined in the Materials and Methods. Control samples were age and sex matched to injured groups. Median head proportion of NISS was lower in the severe than the moderate TBI group due to greater severity of extra-cerebral trauma. 

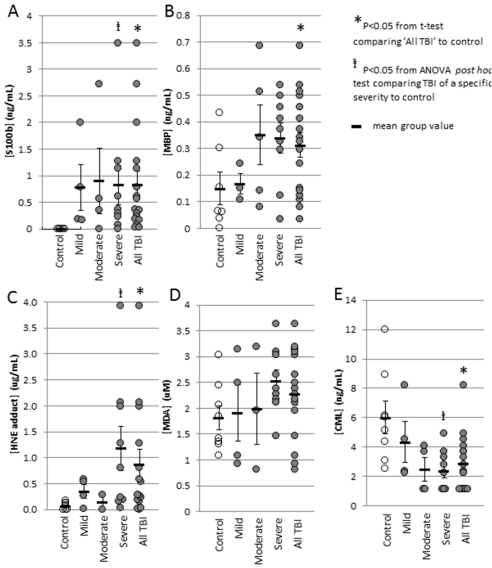